\title{
Tendon-bone contact pressure and biomechanical evaluation of a modified suture-bridge technique for rotator cuff repair
}

\author{
Mike H. Baums • Michael Geyer • Meike Büschken • \\ Gottfried H. Buchhorn - Gunter Spahn • \\ Hans-Michael Klinger
}

Received: 2 June 2009/Accepted: 14 September 2009/Published online: 14 October 2009

(C) The Author(s) 2009. This article is published with open access at Springerlink.com

\begin{abstract}
The aim of the study was to evaluate the timezero mechanical and footprint properties of a suture-bridge technique for rotator cuff repair in an animal model. Thirty fresh-frozen sheep shoulders were randomly assigned among three investigation groups: (1) cyclic loading, (2) load-to-failure testing, and (3) tendon-bone interface contact pressure measurement. Shoulders were cyclically loaded from 10 to $180 \mathrm{~N}$ and displacement to gap formation of 5- and 10-mm at the repair site. Cycles to failure were determined. Additionally, the ultimate tensile strength and stiffness were verified along with the mode of failure. The average contact pressure and pressure pattern were investigated using a pressure-sensitive film system. All of the specimens resisted against 3,000 cycles and none of them reached a gap formation of $10 \mathrm{~mm}$. The number of cycles to 5 -mm gap formation was $2,884.5 \pm 96.8$ cycles. The ultimate tensile strength was $565.8 \pm 17.8 \mathrm{~N}$ and stiffness was $173.7 \pm 9.9 \mathrm{~N} / \mathrm{mm}$. The entire specimen presented a unique mode of failure as it is well known in using high strength sutures by pulling them through the tendon. We observed a mean contact pressure of $1.19 \pm 0.03 \mathrm{MPa}$, applied on the
\end{abstract}

\footnotetext{
M. H. Baums ( $₫) \cdot$ M. Büschken · G. H. Buchhorn ·

H.-M. Klinger

Department of Orthopaedic Surgery,

University of Göttingen Medical Centre (UMG),

Georg-August-University, Robert-Koch-Str. 40,

37099 Göttingen, Germany

e-mail: mbaums@t-online.de

M. Geyer

Department of Orthopaedic Surgery,

St. Vinzenz Klinik Pfronten, Pfronten, Germany

G. Spahn

Clinic of Traumatology and Orthopaedic Surgery,

Eisenach, Germany
}

footprint area. The fundamental results of our study support the use of a suture-bridge technique for optimising the conditions of the healing biology of a reconstructed rotator cuff tendon. Nevertheless, an individual estimation has to be done if using the suture-bridge technique clinically. Further investigation is necessary to evaluate the cell biological healing process in order to achieve further sufficient advancements in rotator cuff repair.

Keywords Rotator cuff repair - Contact pressure . Suture-bridge $\cdot$ Sheep shoulder

\section{Introduction}

Sufficient healing after open or arthroscopic rotator cuff repair still remains one of the challenges in shoulder surgery. Despite an improvement in the surgical technique, persistent tear rates have been reported [4, 13, 14]. Gerber et al. [15] suggested that the repair should provide high initial fixation strength, allowing for only minimal gap formation between the tendon and its insertion site. In addition, it is postulated that re-establishing the anatomical footprint of the tendonbone insertion is a key factor to facilitate healing of the tendon to the bone [1]. The integrity of the repair site, in particular, has been shown to correlate with clinical improvement, particularly the return of strength $[4,13]$. Therefore, the goal is to reconstruct the footprint configuration accompanied by the restoration of its mechanical performance in order to establish an adequate environment for optimal healing of the tendon to the bone.

The native footprint area could be recreated more accurately with the use of the double-row technique when compared to a single-row of anchors [3, 26, 29]. Additionally, previous studies were able to demonstrate a higher 
initial mechanical strength of the double-row repair by means of isometric cyclic loading at time zero $[2,18,21$, 22]. Nonetheless, recent trials have not been able to confirm these results for clinical applications $[10,12,16]$.

Recently, the suture-bridge technique was introduced to maximise the utility of a single-row construct by using the suture limbs from the medial mattress sutures to bridge and compress the repaired tendon [24]. The suture-bridge technique is postulated to restore the footprint contact area and unite the advantages of repair with those of suture anchor and transosseous repair [11, 24]. Thus, there is still no consensus as to which procedure, i.e. suture anchors or suture-bridge fixation, will provide the optimal conditions for cell biological tendon-to-bone healing [1]. Furthermore, there is still interest in developing a strong repair construct.

Given this current situation in the treatment of rotator cuff injury, the purpose of this study was to determine the initial mechanical properties in cyclic loading and load-tofailure as well as the initial contact pressure over a defined rotator cuff footprint for a modified suture-bridge technique, using a titanium anchor screw. The study was conducted with the hypothesis that the presented technique exceeds the clinically relevant $250-\mathrm{N}$ load threshold and would resist an isometric load of 3,000 cycles.

\section{Materials and methods}

Thirty sheep shoulders (specimen age, 2 years) were harvested fresh, wrapped in saline-soaked gauze and stored frozen at $-20^{\circ} \mathrm{C}[2,3,31]$. Shoulders were dissected from all of the soft tissues except for the infraspinatus muscle and tendon. No pre-existing rotator cuff abnormalities were noted in any of the specimens. The infraspinatus tendon was sharply detached from its insertion site to mimic a standardised full-thickness tear. Right and left shoulders were randomly assigned among three investigation groups that were based on the use of a special titanium anchor screw (LASA-DR-Screw ${ }^{\circledR}$, KÖNIGSEE Implantate, Aschau, Germany): (1) cyclic loading, (2) load-to-failure testing and (3) tendon-bone interface contact pressure measurement. All of the repairs were coupled with braided nonabsorbable polyethylene suture sized USP No. 2 ( $\mathrm{HiFi}^{\circledR}$; ConMed Linvatec, Largo, FL, USA).

The LASA-DR-Screw ${ }^{\circledR}$ (Fig. 1) consists of a pinpoint to fix the medial row of sutures, a threaded rod to assure anchorage of the screw to the bone, a thread-free neck and a flat head. Using a freehand technique, a medial bone channel is made with a punch at the medial footprint border, with a lateral tilt of $70-80^{\circ}$ inclination towards the footprint area (Fig. 2a). After that a punch is inserted through the cortex of the lesser tuberosity in parallel direction to the footprint surface (Fig. 2a). Then the length

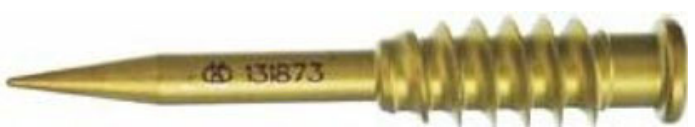

Fig. 1 LASA-DR-Screw ${ }^{\circledR} \quad$ (KÖNIGSEE Implantate, Aschau, Germany) demonstrating the pinpoint to fix the medial row of sutures, the threaded rod to assure anchorage of the screw to the bone, the thread-free neck and the flat head

of the screw is determined, according to the length of the footprint between the tuberosity and the medial footprint border. Great care is taken not to perforate the humeral cartilage. Two sutures are then inserted around the tip of the screw using a guiding device (Fig. 2b). After perforating the sutures medially through the tendon, they were secured by arthroscopic Mason-Allen stitches [28]. The sutures were then tensioned, crossed over the tendon's footprint, tied around the screw neck and secured by the flat head (Fig. 2c-h). Stitches were first tied with the use of a sliding double half-hitch knot, and then secured by a series of four reversing half hitches on alternative posts. To standardise tension for the repair, no less than $4 \mathrm{~kg}$ of tensile force was used to secure each knot. This was measured by a tensiometer. Dissections, preparations, repairs and experimental testing were performed by one single-experienced shoulder surgeon after thawing the shoulders for $24 \mathrm{~h}$ at room temperature.

\section{Cyclic load testing}

Examinations were performed using a material-testing machine [Zwick 1445, Zwick-Roell, Ulm, Germany]. The data were recorded with the dedicated software [testXpert 12, Zwick-Roell, Ulm, Germany] and a load-displacement curve. The proximal end of the infraspinatus tendon was set in a tendon clamp, leaving approximately $6 \mathrm{~cm}$ between the clamp and the site of repair. To prevent it from slipping out of the clamp during testing, "cryo-jaws" for soft tissue fixation were used [2, 19]. The humerus was fixed in a custom rig designed to evenly distribute loads across the tendon. To simulate postoperative conditions, a cyclic loading was performed, similar to previous studies $[2,8$, 27]. Shoulders were loaded in the physiologic direction of the rotator cuff tendon, perpendicular to the longitudinal axis of the humerus, as previously described [2, 19, 27]. The loading force was applied with the humerus maintained at a constant angle relative to the tendon, resulting in simulated isometric muscle contraction. After pretension to $10 \mathrm{~N}$ for $1 \mathrm{~min}$, each construct was cyclically loaded to 3,000 cycles from 10 to $180 \mathrm{~N}$ with a 5 -s cycle $[2,7,8$, 19]. These parameters have been reported as the physiologic loads and speeds that occur in normal daily activity and were therefore considered the best manner to simulate the postoperative condition $[8,27]$. The tests were stopped 

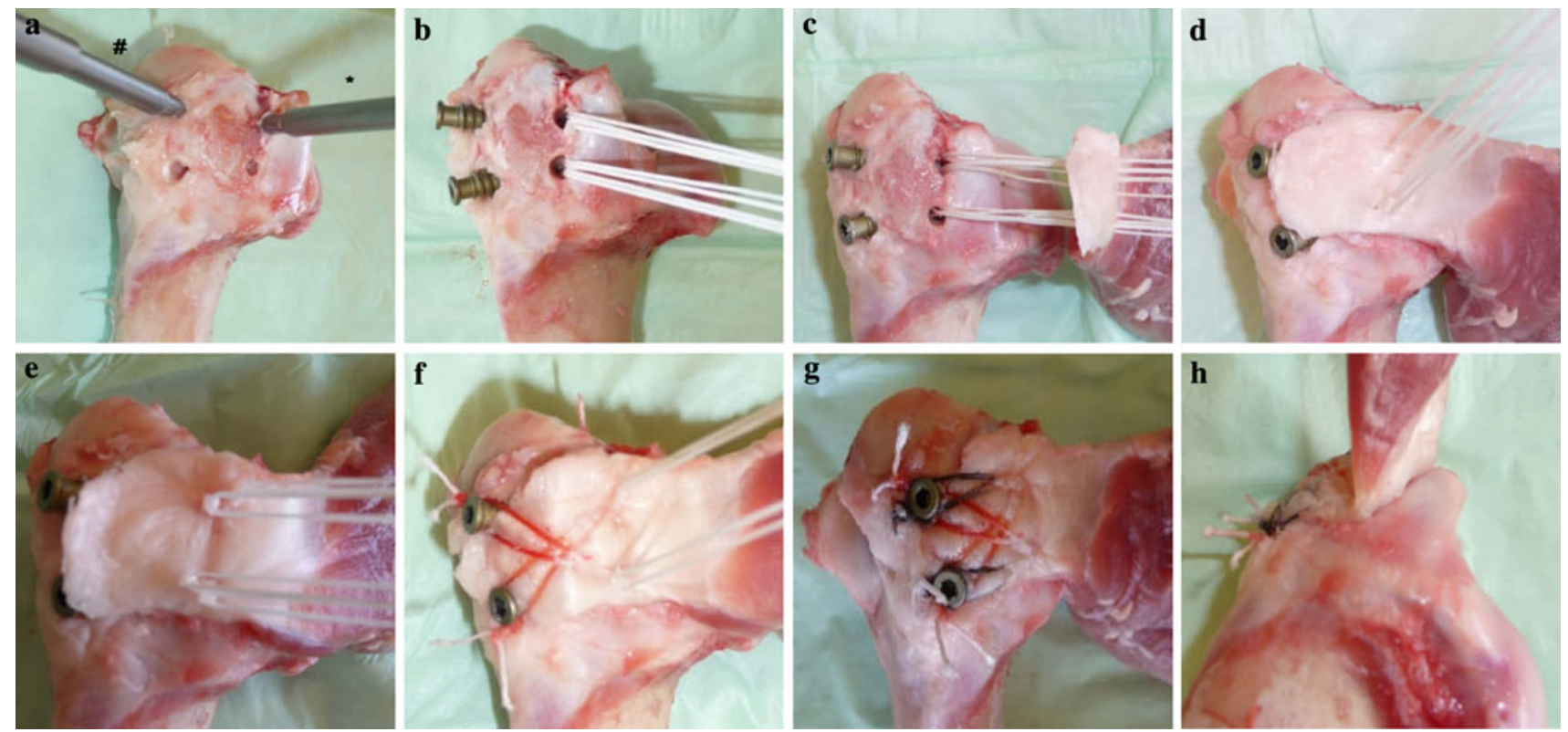

Fig. 2 A medial bone channel is made with a punch (*) at the medial footprint border (a). A suturing instrument is equipped with a shuttle suture and brought into this bone channel. A punch (\#) is then inserted through the lateral cortex of the lesser tuberosity. Then, the screw is placed in parallel to the footprint, with two sutures inserted around the

screw pinpoint (b). The tendon is medially fixed by arthroscopic Mason-Allen stitches (c-e). Sutures were tensioned over the tendon's footprint, then tied around the screw neck, and secured by the flat head $(\mathbf{f}-\mathbf{h})$

when complete failure (e.g. repair site gap $10 \mathrm{~mm}$, defect of the tendon-bone construct) or a total of 3,000 cycles was attained. Gap formation at each repair site was measured using an extensometer. The number of cycles creating a 5-mm and 10-mm gap was recorded along with the mechanism of failure $[2,19,27]$.

\section{Load-to-failure testing}

With use of a material-testing machine [Zwick 1445, ZwickRoell, Ulm, Germany], a pretension of $10 \mathrm{~N}$ was applied and the clamp and custom rig were checked for tightness before beginning the load-to-failure testing. The load $[\mathrm{N}]$ and the displacement $[\mathrm{mm}]$ were digitally recorded by a deformation curve, and the mode of failure was documented. Tests were stopped when complete failure was attained (e.g. repair site gap of $10 \mathrm{~mm}$, defect of the tendon-bone construct). A load of $250 \mathrm{~N}$ was chosen as the minimum clinically relevant ultimate failure load, based on previous studies of rotator cuff fixation techniques $[6,7,15]$.

\section{Contact pressure testing}

A pressure-sensitive film (Prescale film, Super Low Pressure type, Fuji Photo Film Co Ltd, Tokyo, Japan) was used to measure the interface contact pattern and pressure between the infraspinatus tendon and the insertion site $[3,24,29]$.

The pressure-sensitive film was cut in a standardised fashion for all specimens to conform to the $1 \times 2 \mathrm{~cm}$ footprint defect. Then, the film was placed under a prepared template so that we were able to prepare holes on the film in a uniform pattern. The film was sealed in an impermeable polyethylene sheet and inserted between the tendon-bone interfaces after insertion of the screws and sutures, while great care was taken to keep the film dry by constantly using gauze to absorb the moisture from the tendon to the bone $[3,24,29]$. The sutures connecting the tendon to the bone were carefully passed through the prepared holes. These measures were conformed so as to attain the best possible panoramic view of the contact pressure and pressure pattern. After the repair, the film was left in place for $2 \mathrm{~min}$, as recommended by the manufacturer. Sutures were then cut, the film was scanned into a Fuji Film Prescale Pressure Densitometer (FDP-305E, Fuji Photo Film Co Ltd, Tokyo, Japan), and the average contact pressure and pressure pattern were detected.

\section{Results}

All of the specimens resisted against 3,000 cycles. None of them reached a gap formation of $10 \mathrm{~mm}$. The number of cycles to 5-mm gap formation was 2,884.5 \pm 96.8 cycles. There was no screw-related failure during cyclic loading. Humeral cartilage was not perforated in all of the specimens. 
The ultimate tensile strength of the suture-bridge technique was $565.8 \pm 17.8 \mathrm{~N}$ and stiffness was $173.7 \pm$ $9.9 \mathrm{~N} / \mathrm{mm}$. The entire specimen presented a previously described unique mode of failure as it is well known in using high strength sutures by pulling them through the tendon $[2,5,20]$. Suture breakage was not observed. There was no screw-related failure during load-to-failure testing as well.

We detected a mean contact pressure of $1.19 \pm$ $0.03 \mathrm{MPa}$ applied on the footprint area. However, the pressure around the insertion of the knots was higher than in the area between the knots. Overall, the suture-bridge technique produced a homogenous pattern throughout the tendon-bone contact area.

\section{Discussion}

The most important findings of the study are excellent biomechanical and tendon-bone-pressure characteristics of the presented modified suture-bridge technique. We confirmed our hypothesis, with the principle that this technique exceeds the clinically relevant $250-\mathrm{N}$ load threshold as well as resisted against an isometric load of 3,000 cycles. Additionally, the contact pressure over a defined footprint area is comparable to the results of a double-row technique [3, 24, 29].

Several studies have demonstrated the mechanical characteristics of different techniques for rotator cuff repair, mainly dealing with the use of suture anchor systems. These studies have reported excellent mechanical behaviour for the double-row repair [2, 18, 21, 22]. Nonetheless, its clinical superiority remains controversial. In prospective surveys, the double- and single-row techniques provided comparable clinical and MRI arthrography short-term outcomes [12], especially in patients with small and medium rotator cuff tears $(<3 \mathrm{~cm})$ [23]. Only Charousset et al. [10] demonstrated significantly better tendon healing results for the double-row technique, in a computed tomographic arthrography follow-up.

Despite its widespread use, our review of the literature revealed only a few papers examining clinical use of the suture-bridge technique [13], in basic trials [6, 9, 24] or presented as a technical note $[11,25]$. In a recent study, Frank et al. [13] confirmed a healing rate of $88 \%$ with MRI studies, as well as excellent clinical function after a mean follow-up of 14.6 months. The authors concluded that the suture-bridge technique could protect the biological environment of tendon-to-bone healing over a short-term period. Burkhead et al. [6] simulated a suture-bridge technique repair with a combination of transosseous sutures and suture anchor systems in cadaver shoulder specimen. They used a monotonic loading regime and found a mean failure of the repair at $404 \mathrm{~N}$. In comparison with transosseous and single-row techniques, the repair failed at 55-67\% higher loads, respectively. A cyclic loading test was not performed (Table 1). Comparing the mean load-to-failure result of $565.81 \mathrm{~N}$ to a recent study evaluating the differences between single- and double-row techniques [2], we were able to confirm higher failure loads, as shown by Burkhead et al. [6]. Certainly, comparing these results to the mentioned study [6], it has to be considered that we used an animal model and the infraspinatus tendon instead of the human supraspinatus tendon.

Busfield et al. [9] conducted a biomechanical comparison of two suture-bridge repair techniques with and without medial row knots using metallic suture anchors and PushLocks (Arthrex, Naples, FL, USA). The authors confirmed the necessity of medial row knots, in order to preserve the integrity of the repair construct and reach higher ultimate failure loads of up to $352.9 \mathrm{~N}$ (Table 1).

The average number of cycles to $5-\mathrm{mm}$ gap formation in the study at hand was $2,884.5$ cycles. The entire specimen resisted 3,000 cycles. Comparing these results to studies using an analogue experimental set-up, it seems that the suture-bridge technique provides strength superior to that of a single-row, double-row and transosseous techniques $[2,19]$. The contact pressure at the tendon-bone interface was the same when comparing this technique to a doublerow technique using a combination of arthroscopic MasonAllen stitches and horizontal mattress stitches [3]. When using a combination of simple and horizontal mattress stitches, contact pressure was reduced [3]. In summary, the use of a suture-bridge technique maintained a homogenous pressure pattern at the tendon-bone interface, as supported by the results of Park et al. [24].

The entire tested specimen in the current study failed by pulling the suture through the tendon when the maximum load was reached, as noticed in former investigations [2, 5, 20]. Nevertheless, the maximum load was significantly higher compared to a double-row repair that was tested in a prior study, with the use of braided polyblend polyethylene sutures as well as with braided polyester sutures [2]. The problem of early suture breakage seems to be solved by the improved material properties of the new high-performance suture material. However, it has to be noted that the more rigid characteristics of the new braided polyblend polyethylene suture material $[20,32]$ can more easily cut in a parallel direction through the defective tendon, when used in healthy rotator cuff specimens [2, 5, 20]. This aspect is particularly important, because most of the torn human rotator cuff tendons are degenerated. Tendons that fail a single-row repair are typically grade III degenerate tendons, according to the classification of Goutallier et al. [17]. In the recent study, failure of the repair is indeed due to suture pulling through the tendon, resulting in a 
Table 1 Summary of the results of current studies investigating the suture-bridge or suture-bridge-like repair techniques

\begin{tabular}{|c|c|c|c|c|}
\hline & Experimental setting & Tested technique & Fixation material & Result \\
\hline Burkhead et al. [6] & Load-to-failure & $\begin{array}{l}\text { TOS versus SR } \\
\text { versus SB }\end{array}$ & $\begin{array}{l}\text { TOS: } 3 \text { Ethibond }{ }^{\circledR} \text { suture } \\
\text { SR: } 3 \text { metallic anchor systems } \\
\text { SB: PANALOK }{ }^{\circledR} \text { anchor system }\end{array}$ & $\mathrm{SB}>\mathrm{TOS}>\mathrm{SR}$ \\
\hline Busfield et al. [9] & $\begin{array}{l}\text { Cyclic loading } \\
\text { Load-to-failure }\end{array}$ & $\begin{array}{l}\text { DRSB1 versus } \\
\text { DRSB2 }\end{array}$ & $\begin{array}{l}\text { DR-SB1: } 2 \text { metallic anchor systems medial, } \\
2 \text { PushLocks }{ }^{\circledR} \text { lateral } \\
\text { DR-SB2: } 2 \text { metallic anchor systems medial, } \\
2 \text { PushLocks }{ }^{\circledR} \text { lateral }\end{array}$ & DRSB2 $>$ DR-SB 1 \\
\hline Park et al. [24] & $\begin{array}{l}\text { Contact pressure } \\
\text { Contact area }\end{array}$ & $\begin{array}{l}\text { DR versus SB2 } \\
\text { versus SB4 }\end{array}$ & $\begin{array}{l}\text { DR: } 4 \text { BIO-Corkscrew }{ }^{\circledR} \text { anchor systems } \\
\text { SB2: } 1 \text { BIO-Tenodesis screw }{ }^{\circledR} \\
\text { SB4: } 2 \text { BIO-Tenodesis screw }{ }^{\circledR}\end{array}$ & $\mathrm{SB} 4>\mathrm{SB} 2>\mathrm{DR}$ \\
\hline
\end{tabular}

$D R$ double-row, $D R S B 1$ double-row-suture-bridge group 1 (without medial row knots), DRSB2 double-row-suture-bridge group 2 (with medial row knots), $T O S$ transosseous, $S R$ single-row, $S B$ suture bridge, $S B 2$ suture bridge (2 suture bridges), $S B 4$ suture bridge (4 suture bridges)

considerably higher failure force when compared to double-row repair [2].

We could assume that this phenomenon is due to a steady and homogenous pressure distribution of the tendon-bone construct when using the suture-bridge technique. On the other hand, the contact pattern between the fixation points could be increased by the suture-bridge repair, resulting in more footprint coverage than is observed for single-row techniques [24]. This may be due the characteristics of an 'interconnected' repair, in contrast to the double-row technique that is based on separate fixation points [25]. Additionally, the suture-bridge technique provides a better compression vector when compared with suture anchor techniques [24]. Park et al. [24] analysed the contact area of two different suture-bridge techniques in which a 4-suturebridge technique, similar to that used in our evaluation, yielded a significantly higher contact area and interface pressure than double-row and 2-suture-bridge techniques (Table 1). The authors conclude that this technique may lead to further improvement in the repair of rotator cuff tears, thus optimising the chances of healing the tendon to the bone. In our study, the mean contact pressure was $1.19 \mathrm{MPa}$, which is comparable to the results of a double-row technique [3].

Our investigation presents some limitations. The sheep infraspinatus tendon was chosen because it has previously been demonstrated to be similar in size, shape and microstructure to the human supraspinatus tendon [15]. But it has to be stated that it is different from the degenerated and thinner supraspinatus tendons seen in human shoulders with chronic rotator cuff tears, as already mentioned earlier. Consequently, it remains an approximation to the human condition and clinically used rotator cuff repair techniques. Nevertheless, this animal model has been used extensively to evaluate rotator cuff tendon repairs in previous studies [2, $3,15,19$ ] and allows interstudy comparison.

Furthermore, although great care was taken to minimise interference when preparing holes in the film, with regard to the pressure measurements, this may have yielded some artefacts. However, it would be impossible to obtain the entire contact pressure distribution and pressure pattern of the investigated techniques if pressure-sensitive film was only inserted between the tendon-bone interfaces. Additionally, using pressure-sensitive film that detects a pressure range between 0.50 and $2.50 \mathrm{MPa}$ may have underestimated the contact area [29].

The results of the current study provide information on the footprint coverage and mechanical strength of a suturebridge technique during the immediate postoperative clinical situation. By passing the sutures medially, the repair takes advantage of healthier tendon tissue to improve fixation strength [25]. Due to the interconnected repair, one is able to achieve a homogenous tendon-to-bone pressure that has been shown to influence healing [30]. Beyond these considerations, the optimal pressure range for the tendon to heal to the bone is not known. Therefore, low pressure may cause separation of the tendon-bone interface, whereas high pressure may affect the vascularisation of the tendon and result in impaired tissue healing. Thus, it should be noted that our evaluation does not address the influence of healing and/or remodelling on the strengths and footprint coverage of the repair. After comparing our results with recent reports in the literature [2, 3, 19, 21, 22, 24, 26, 29], the suture-bridge technique seems to be a further improvement in rotator cuff surgery [13, 24-26]. Therefore, the presented technique may lead to clinical results superior to several double-row fixations, because it offers biomechanical advantages with low gap formation in cyclic loading and a uniform tendon-bone-contact pressure.

\section{Conclusion}

In conclusion, the fundamental results of our study (i.e. high mechanical load resistance, high contact pressure and 
contact area) support the use of a suture-bridge technique for reconstruction of a torn rotator cuff tendon. Nevertheless, an individual estimation has to be done when using the suture-bridge technique clinically as suggested by Park et al. [25]. A biomechanical comparison of a double-row versus the presented suture-bridge technique may be helpful but finally, in vivo trials are necessary to gain information on the cell biological healing process of suture-bridge techniques, to emphasise potential biological advantages and to accomplish more sufficient advancements in rotator cuff repair.

Conflict of interest statement The authors declare that they have no conflict of interest.

Open Access This article is distributed under the terms of the Creative Commons Attribution Noncommercial License which permits any noncommercial use, distribution, and reproduction in any medium, provided the original author(s) and source are credited.

\section{References}

1. Apreleva M, Özbaydar M, Fitzgibbons PG, Warner JJP (2002) Rotator cuff tears: the effect of the reconstruction method on three-dimensional repair site area. Arthroscopy 18:519-526

2. Baums MH, Buchhorn GH, Spahn G, Poppendieck B, Schultz W, Klinger HM (2008) Biomechanical characteristics of single-row repair in comparison to double-row repair with consideration of the suture configuration and suture material. Knee Surg Sports Traumatol Arthrosc 16:1052-1060. doi:10.1007/s00167-008-0590-2

3. Baums MH, Spahn G, Steckel H, Fischer A, Schultz W, Klinger HM (2009) Comparative evaluation of the tendon-bone interface contact pressure in different single- versus double-row suture anchor repair techniques. Knee Surg Sports Traumatol Arthrosc. doi:10.1007/s00167-009-0771-7

4. Bishop J, Klepps S, Lo IK, Bird J, Gladstone JN, Flatow EL (2006) Cuff integrity after arthroscopic versus open rotator cuff repair: a prospective study. J Shoulder Elbow Surg 15:290-299

5. Bisson LJ, Manohar LM, Wilkins RD, Gurske-Deperio J, Ehrensberger MT (2008) Influence of suture material on the biomechanical behaviour of suture-tendon specimens. Am J Sports Med 36:907-912

6. Burkhead WZ, Skedros JG, O'Rourke PJ, Pierce WA, Pitts TC (2007) A novel double-row rotator cuff repair exceeds strengths of conventional repairs. Clin Orthop Rel Res 461:106-113

7. Burkhart SS, Diaz Pagan JL, Wirth MA, Athanasiou KA (1997) Cyclic loading of anchor-based rotator cuff repairs: confirmation of the tension overload phenomenon and comparison of suture anchor fixation with transosseous fixation. Arthroscopy 13:720 724

8. Burkhart SS, Johnson TC, Wirth MA, Athanasiou KA (1997) Cyclic loading of transosseous rotator cuff repairs: tension overload as a possible cause of failure. Arthroscopy 13:172-176

9. Busfield BT, Glousman RE, McGarry MH, Tibone JE, Lee TQ (2008) A biomechanical comparison of 2 technical variations of double-row rotator cuff fixation. Am J Sports Med 36:901-906

10. Charousset C, Grimberg J, Duranthon LD, Bellaiche L, Petrover D (2007) Can a double-row anchorage technique improve tendon healing in arthroscopic rotator cuff repair? A prospective, nonrandomized, comparative study of double-row and single-row anchorage techniques with computed tomography arthrography tendon healing assessment. Am J Sports Med 35: 1247-1253

11. Franceschi F, Longo UG, Ruzzini L, Ribello G, Mafulli N, Denaro V (2007) The Roman Bridge: a "double pulley-suture bridges" technique for rotator cuff repair. BMC Musculoskelet Disord 8:123-129

12. Franceschi F, Ruzzin L, Longo UG, Martina FM, Zobel BB (2007) Equivalent clinical results of arthroscopic single-row and double-row suture anchor repair for rotator cuff tears. Am J Sports Med 35:1254-1260

13. Frank JB, ElAtrache NS, Dines JS, Blackburn A, Crues J, Tibone JE (2008) Repair site integrity after arthroscopic transosseousequivalent suture-bridge rotator cuff repair. Am J Sports Med 36:1496-1503

14. Galatz LM, Ball CM, Teefey SA, Middleton WD, Yamaguchi K (2004) The outcome and repair integrity of completely arthroscopically repaired large and massive rotator cuff tears. J Bone Joint Surg Am 86-A:219-224

15. Gerber C, Schneeberger AG, Beck M, Schlegel U (1994) Mechanical strength of repairs of the rotator cuff. J Bone Joint Surg Br 76-B:371-380

16. Grasso A, Milano G, Salvatore M, Falcone G, Deriu L, Fabbriciani C (2009) Single-row versus double-row arthroscopic rotator cuff repair: a prospective randomized clinical study. Arthroscopy 25:4-12

17. Goutallier D, Postel JM, Bernageau J, Lavau L, Voisin MC (1994) Fatty muscle degeneration in cuff ruptures. Pre- and postoperative evaluation by CT scan. Clin Orthop Relat Res 304:78-83

18. Kim DH, ElAttrache NS, Tibone JE, Jun BJ, DeLaMora SN, Kvitne RS, Lee TQ (2005) Biomechanical comparison of a single-row versus double-row suture anchor technique for rotator cuff repair. Am J Sports Med 34:407-414

19. Klinger HM, Steckel H, Spahn G, Buchhorn GH, Baums MH (2007) Biomechanical comparison of double-loaded suture anchors using arthroscopic Mason-Allen stitches versus traditional transosseous suture technique and modified Mason-Allen stitches for rotator cuff repair. Clin Biomech 22:106-111

20. Kowalsky MS, Dellenbaugh SG, Erlichman DB, Gardner TR, Levine WN, Ahmad CS (2008) Evaluation of suture abrasion against rotator cuff tendon and proximal humerus bone. Arthroscopy 24:329-334

21. Ma CM, Comerford L, Wilson J, Puttlitz CM (2006) Biomechanical evaluation of arthroscopic rotator cuff repairs: doublerow compared with single-row fixation. J Bone Joint Surg Am 88-A:403-410

22. Milano G, Grasso A, Zarelli D, Deriu L, Cillo M, Fabbriciani C (2008) Comparison between single-row and double-row rotator cuff repair: a biomechanical study. Knee Surg Sports Traumatol Arthrosc 16:75-80

23. Park JY, Lhee SH, Choi JH, Park HK, Yu JW, Seo JB (2008) Comparison of the clinical outcomes of single- and double-row repairs in rotator cuff tears. Am J Sports Med 36:1310-1316

24. Park MC, ElAttrache NS, Tibone JE, Ahmad CS, Jun BJ, Lee TQ (2007) Part I: Footprint contact characteristics for a transosseousequivalent rotator cuff repair technique compared with a doublerow technique. J Shoulder Elbow Surg 16:461-468

25. Park MC, ElAttrache NS, Ahmad CS, Tibone JE (2006) "Transosseous-equivalent" rotator cuff repair technique. Arthroscopy 22:1360.e1-1360.e5

26. Park MC, Cadet ER, Levine WN, Bigliani LU, Ahmad CS (2005) Tendon-to bone pressure distributions at a repaired rotator cuff footprint using transosseous suture and suture anchor fixation techniques. Am J Sports Med 33:1154-1159 
27. Reed SC, Glossop N, Ogilvie-Harris DJ (1996) Full-thickness rotator cuff tears. A biomechanical comparison of suture versus bone anchor techniques. Am J Sports Med 24:46-48

28. Scheibel MT, Habermeyer P (2003) A modified Mason-Allen technique for rotator cuff repair using suture anchors. Arthroscopy 19:330-333

29. Tuoheti Y, Itoi E, Yamamoto N, Seki N, Abe H, Minagawa H, Okada K, Shimada Y (2005) Contact area, contact pressure, and pressure patterns of the tendon-bone interface after rotator cuff repair. Am J Sports Med 33:1869-1874

30. Weiler A, Hoffmann RFG, Bail HJ, Rehm O, Südkamp NP (2002) Tendon healing in a bone tunnel, part II: histologic analysis after biodegradable interference fir fixation in a model of anterior cruciate ligament reconstruction in sheep. Arthroscopy 18:124-135

31. Woo SL, Orlando CA, Camp JF, Akeson WH (1986) Effects of post-mortem storage by freezing on ligament tensile behaviour. J Biomech 19:399-404

32. Wüst DM, Meyer DC, Favre P, Gerber C (2006) Mechanical and handling properties of braided polyblend polyethylene sutures in comparison to braided polyester and monofilament polydioxanone sutures. Arthroscopy 22:1146-1153 